\title{
NEUTRALISATION OF THE LEAR ELECTRON-COOLING BEAM: EXPERIMENTAL RESULTS
}

\author{
J. Bosser, F. Caspers, M. Chanel, R. Ley, R. Maccaferri, S. Maury,
} I. Meshkov*, G. Molinari, V. Polyakov*, A. Smirnov*, O. Stepashkin*, E. Syresin*, G. Tranquille, and F. Varenne

\begin{abstract}
The LEAR electron cooler uses dense electron beams. Because of this high density the large space-charge potential induces significant drawbacks for the cooling process itself. As a consequence a space-charge neutralisation system has been implemented on the cooler. It consists of two positively polarised pairs of electrodes, placed on the outside of the drift space, which aim to store the ionised positive ions of charge $Z$. The major difficulty arises in the neutralisation instabilities [1]. This paper reports on the technological aspects, the measurement techniques and the results.
\end{abstract}

*JINR, Dubna, Russia

Paper presented at the 1995 Particle Accelerator Conference Dallas, USA, 30 April - 5 May 1995

Geneva, Switzerland

$10 / 5 / 95$ 


\title{
NEUTRALISATION OF THE LEAR ELECTRON-COOLING BEAM: EXPERIMENTAL RESULTS
}

\author{
J. Bosser, F. Caspers, M. Chanel, R. Ley, R. Maccaferri, S. Maury, \\ G. Molinari, G. Tranquille, and F. Varenne \\ CERN, CH-1211 Geneva 23
}

\section{Meshkov, V. Polyakov, A. Smirnov, O. Stepashkin, and E. Syresin JINR, Dubna, Russia}

The LEAR electron cooler uses dense electron beams. Because of this high density the large space-charge potential induces significant drawbacks for the cooling process itself. As a consequence a space-charge neutralisation system has been implemented on the cooler. It consists of two positively polarised pairs of electrodes, placed on the outside of the drift space, which aim to store the ionised positive ions of charge $Z$. The major difficulty arises in the neutralisation instabilities [1]. This paper reports on the technological aspects, the measurement techniques and the results.

\section{INTRODUCTION}

In the frame of the electron cooling (ECOOL) it is worth having at our disposal large electron beam intensities, $I_{b}$. In such a case, mainly for low-velocity beams $(\beta=v / c$ $\ll 1)$, the space-charge effects induce some drawbacks.

In the drift space, at ground potential, where the electrons and the ions to be cooled are merged, the spacecharge potential $U_{s p}[\mathrm{~V}]$ as a function of the radius $r$ is given by (Fig. 1a,b):

$$
U_{s p}(r) \equiv-\frac{30 I_{b}}{\beta}(1-\eta)\left[1+2 \ln \left(\frac{b}{a}\right)-\left(\frac{r}{a}\right)^{2}\right], \quad r \leq a(1)
$$

where:

$a, b$ are the e-beam and beam-pipe radii respectively,

$\eta=Z n_{i} / n_{e}=$ stored-ion density/electron-beam density

$=$ neutralisation factor

$I_{b}(A)$ is the electron-beam current.

Therefore the radial electrical field

$$
E_{r}=-\frac{\partial U_{s p}}{\partial r}=-\frac{60 I}{\beta}(1-\eta) \frac{r}{a^{2}}, \quad r \leq a
$$

will induce a transverse force on the electron at radius $r$. This force, though compensated to a certain extent by the longitudinal magnetic field $B_{0}$, will tend to increase the ebeam transverse velocity spread. Even partial neutralisation $(0<\eta<1)$ will reduce this unwanted force.

Another difficulty stems from the fact that, the electron kinetic energy is given by

$$
E_{c}=(\gamma-1) m_{e} c^{2}=e\left(U_{c}+U_{s p}\right) \cong \frac{1}{2} m_{e} v_{e}^{2}
$$

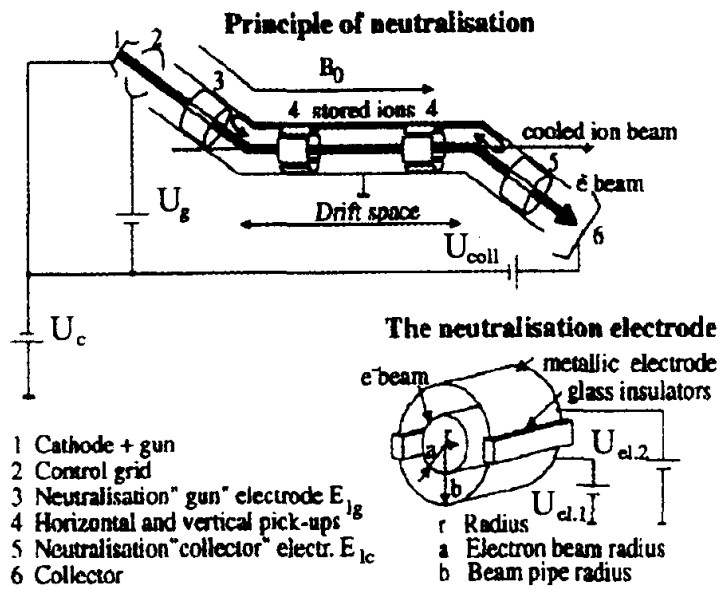

Figure 1: a) ECOOL principle, b) Neutralisation electrode. [where $-U_{c}$, the cathode potential (Fig. 1a)], is a function of the radius $r$. Defining the nominal velocity $v_{0}$ (which is equal to the average velocity of the cooled ions) as

$$
v_{0}=\sqrt{\frac{2 e}{m_{e}}\left(U_{c}+U_{s p}(r=0)\right)}
$$

then

$$
v_{e}(r) \cong v_{0}\left[1+\frac{1}{2} \frac{U_{s p}(r)-U_{s p}(r=0)}{U_{c}+U_{s p}(r=0)}\right]
$$

has the parabolic shape represented in Fig. 2. In the drift space where the dispersion function of the ion storage ring is $D$ and the betatron function $\beta_{\mathrm{H}}$ the ion velocity is given by

$$
v_{i}\left(x_{i}\right) \cong v_{0}\left[1+\frac{d v_{i(l)}}{v_{0}}\right]
$$

with $x_{i}$ the mean horizontal position of an ion with momentum deviation $\Delta p_{i}$

$$
x_{i}=D \frac{\Delta p_{i}}{p}=D \gamma^{2} \frac{d v_{i(l)}}{v_{0}},
$$

The horizontal velocity distribution is shown in Fig. 2. It must be noted that to the mean horizontal position the betatron oscillation has to be added. At LEAR with $D=3.5 \mathrm{~m}, \beta_{\mathrm{H}}=2 \mathrm{~m}$ for $\beta=0.1, I_{e}=0.5 \mathrm{~A}, \eta=0$, the two velocities $v_{e}$ and $v_{i}$ intersect at $x_{i} \cong 2 \mathrm{~mm} \ll$ a such that relatively large emittance ion beams can be heated rather than cooled. 
It is therefore essential either to reduce the dispersion $(D \rightarrow$ 0 ) at the cooler and/or to neutralise the e-beam such that the electron velocity becomes independent of its radius $\left(\eta \rightarrow 1 \Rightarrow U_{s p}(r) \rightarrow 0\right)$.

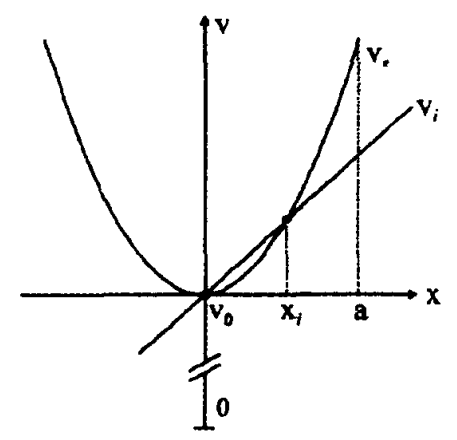

$\mathrm{v}$, electron velocity $v_{i}$ ion velocity $x$ horizontal position $\mathrm{x}_{i}$ ion horiz. position

Figure 2: Electron and ion velocity versus horizontal position.

\section{PRINCIPLE OF NEUTRALISATION $[2,3]$}

For the purpose of neutralisation, two neutralisation electrodes, $E l_{g}$ and $E l_{c}$, consisting of two metallic half cylinders separated by a high-resistive-glass insulator (Fig. 1b), have been installed outside the drift space (Fig. 1a). They are polarized by independent positive power supplies named $U_{e l 1}$ and $U_{e l 2}\left(0 \leq U_{e l}\right.$ s $\left.\leq 6 \mathrm{kV}\right)$. Usually, the voltages on opposite electrodes are not equal such that a transverse $E$-field also exists.

On their way from the cathode to the collector the energetic primary electrons, $e_{1}$, will ionize the residual gas molecules. The ionized low energy, ions, $i_{2}$, and electrons, $e_{2}$, will be submitted to the space charge and to the longitudinal magnetic field forces. They will move towards the cathode or the collector. At the level of the neutralisation electrodes $\left(E l_{g}\right.$ or $\left.E l_{c}\right)$ the ions, $i_{2}$, will be reflected, and therefore stored, whilst the low-energy ionized electrons, $e_{2}$ which drift in the crossed electric and magnetic fields will be collected on the glass insulators. Consequently, the ion density $n_{i}$, and therefore $\eta$, will increase with time.

It should be mentioned that in the case of LEAR the beam-chamber diameter, at the level of $E l_{g}$ and $E l_{c}$, is $100 \mathrm{~mm}$ while in the drift space: $b=140 \mathrm{~mm}$. This will induce a "natural neutralisation" due to the potential well (Eq. 1).

\section{PHYSICAL CONSIDERATIONS}

Many physical issues are the subject of intense studies, e.g.

- The physical and mathematical description of the evolution of $\eta$ versus time, namely what brings the ion escape rate to be equal to the ionization rate $(0<\eta \leq 1)$.

- The conditions for the neutralisation-factor stability. These are discussed in Ref. [4].
Under certain conditions the neutralisation factor becomes unstable, which is detrimental to the cooling process since this implies an instability in the cooled beam velocity [consider the influence of $\eta$ jumps in Eqs. (4) and (1)]. This instability is also of concern for natural neutralisation.

\section{EXPERIMENTAL RESULTS}

\section{A. Neutralisation factor}

The main parameter to be measured is the neutralisation factor $\eta$ whose final values will depend on many parameters such as the polarisation voltages $U_{e l 1,2,3,4}$, the electron current $I_{b}$, the magnetic field $B_{0}$, etc.

When the ions to be cooled, $i_{1}$, are circulating in LEAR, the signal from a longitudinal Schottky pickup is used. It is displayed on a spectrum analyser at a given harmonic of the revolution frequency $f_{0}$. When the neutralisation is effective, the space-charge potential $U_{s p}(r=$ 0 ) is reduced [Eq. (1)], which entails an increase of the $i_{1}$ velocity. From the correction to be applied to $U_{c}$ in order to retrieve $v_{0}$ [Eq. (3)] the value of $\eta$ can be deduced.

A second way to measure $\eta$ is the time of flight method [3]. A high-frequency signal $(200 \leq f \leq 300 \mathrm{MHz})$ is applied e.g. to $E l_{g}$. This signal slightly modulates the longitudinal or transversal density of the e-beam $i_{1}$. The modulated signal is detected by a pair of electrodes at a distance $L$ from the excitation electrode. The phase difference between signals

$$
\phi=\frac{2 \pi f L}{v_{e}(\eta)}
$$

allows us to deduce $v_{e}$ and therefore the neutralisation factor [Eqs. (3),(1)]. The phase $\phi$ can be calibrated by the previous method with ion beams.

Figure 3a shows a measured value of $\phi$ versus time. The time it takes to reach the final value of $\phi$ is the neutralisation time. The initial jump is thought to be due to the stored electrons which are expelled when the electrodes are polarized.

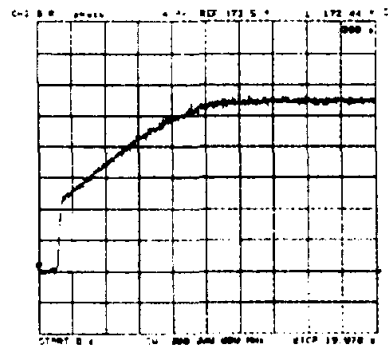

a) Stable $\eta$,

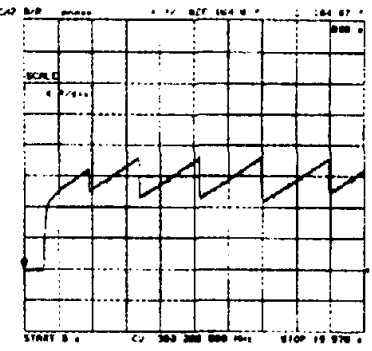

b) Unstable $\eta$
Figure 3: Time of flight or phase $\phi$ measurement versus time.

An unstable neutralisation is easily detected through the observation of the phase discontinuity as in Fig. $3 \mathrm{~b}$. 


\section{B. The Use of Position Pickup Electrodes}

Usually, the pickup electrodes in the drift space (4 in Fig. 1a) are used to measure both the electron $\left(e_{1}\right)$ and cooled ion $\left(i_{1}\right)$ beam horizontal and vertical positions. In the present case, they are used to observe the neutralisation ion (i2) transverse oscillations. Figure $4 a$ shows the transverse oscillations versus time on both pickups during the instability. No longitudinal oscillations have been observed. Figure $4 b$ shows the oscillation frequency distribution. The results of these measurements are coherent with the theoretical computations [4].

The instability occurs simultaneously with a discontinuity in the time-of-flight measurement (Fig. 3b).

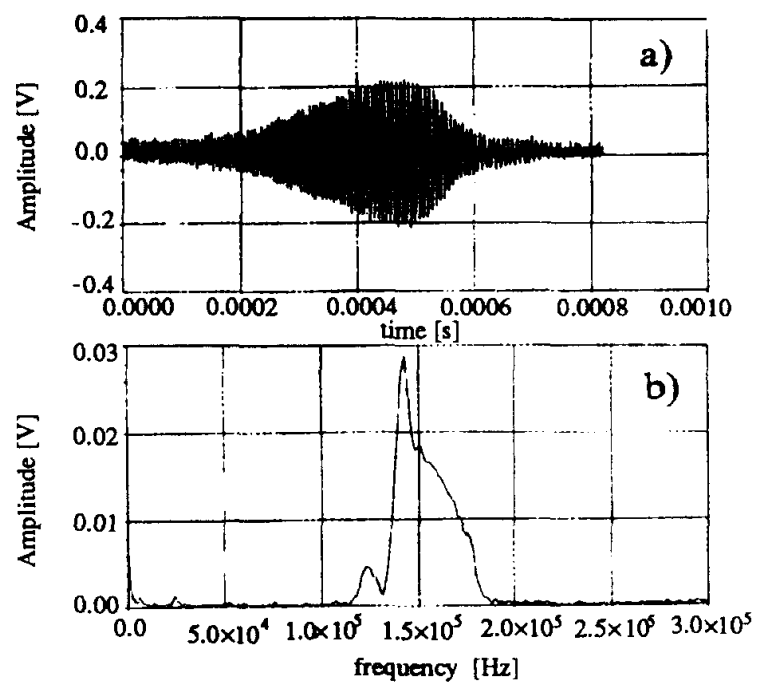

Figure 4: a) Observed instabilities on PUs, b) Frequency distribution.

\section{Attempts for Feedback, the "Shaker"}

In order to damp the neutralisation instabilities, a feedback system has been developed. The signal from one of the position pickups (where the instability is observed), is amplified and phase delayed in such a way as to be applied with the correct phase on an electrode upstream of the measurement pickup. In the present state of experiments, we did not succeed in damping the instabilities. Nevertheless, ions have been "shaken" out of the e-beam in order to render the e-beam velocity stable. An example is given in Fig. 5 where the neutralisation factor $(\eta=1 \Rightarrow \emptyset$ $=100^{\circ}$ ) has been recorded under 4 different conditions. Condition 1: no feedback (shaker) at natural neutralisation, the neutralisation factor is unstable. Condition 2: the shaker is $O N$, the natural neutralisation factor is reduced, but stable. Condition 3: an induced neutralisation is introduced (for $E l g: U_{e l 1}=0 \mathrm{~V}, U_{e l 2}=6 \mathrm{kV}$; for $E l_{c}: U_{e l 1}=0 \mathrm{~V}$, $U_{e l 2}=6 \mathrm{kV}$, see Fig. 1b) while the shaker is still ON. Condition 4: the shaker is OFF while the neutralisation voltages are maintained, the neutralisation factor increases, but becomes unstable.

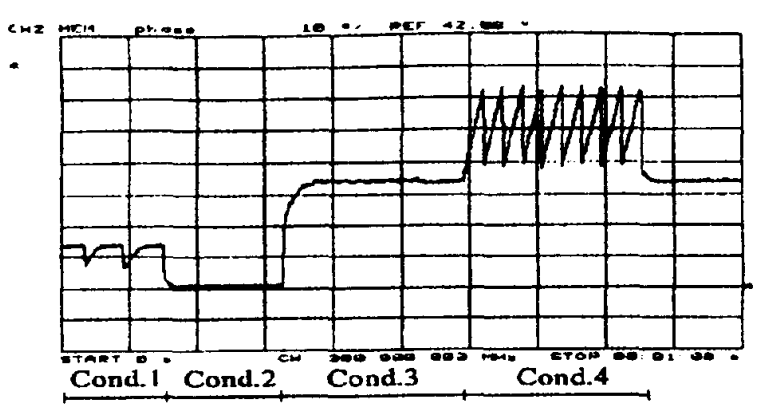

Figure 5: Different uses of the "shaker".

\section{CONCLUSION}

Neutralisation of the electron beam can be achieved. Nevertheless, under certain conditions it becomes unstable. In order to cancel these instabilities a so-called "shaker" if voltage has been applied to one pair of position pick-up electrodes; this reduces but stabilises the neutralisation factor. More investigations and experiments are under way.

\section{REFERENCES}

[1] J. Bosser, R. Ley, I. Meshkov, G. Molinari, V. Polyakov, A. Smimov, E. Syresin, G. Tranquille, and F. Varenne, Electron Cooling with Neutralised Electron Beams, EPAC'94, London, p. 1211.

[2] A.V. Burov, V.I. Kudelainen, V.A. Lebedev, V.V. Parkhomchuk, A.A. Sery, V.D. Shiltsev, Experimental Investigation of an Electron Beam in Compensated State, CERN/PS 93-03 (AR), 1993.

[3] J. Bosser, I. Meshkov, D. Möhl, V.V. Parkhomchuk, E. Syresin, and G. Tranquille, Neutralisation of the LEAR-ECOOL Electron Beam Space Charge, CERN/PS/AR Note 93-08, 1993.

[4] J. Bosser, D. Mohl, F. Varenne, I. Meshkov, E. Syresin, E. Mustafin, P. Zenkevich. Stability conditions for a Neutralised Electron Cooling Beam. This Conference. 
